\title{
Effect of Silicon dioxide coating of acrylic resin surfaces on Candida albicans adhesion
}

\section{Rossano Vinícius Dala Rosa SILVA(a) \\ Maiara Ignácio COSTA ${ }^{(b)}$ \\ Isabele Carrilho JARROS(b) \\ Altair Antoninha DEL BEL CURY(c) \\ Sharanbir Kaur SIDHU(d) \\ Melyssa NEGRI(b) \\ Renata Corrêa PASCOTTO(a)}

(a) Universidade Estadual de Maringá - UEM,

Department of Dentistry, Maringá, PR, Brazil.

(b) Universidade Estadual de Maringá - UEM, Department of Clinical Analysis, Division of Medical Mycology, Maringá, PR, Brazil.

(c) Universidade Estadual de Campinas - Unicamp, Piracicaba Dental School, Department of Prosthodontics and Periodontology, Piracicaba, SP, Brazil.

(d) Queen Mary University of London, Barts \& The London School of Medicine and Dentistry, Centre for Oral Bioengineering, London, UK.

Declaration of Interests: The authors certify that they have no commercial or associative interest that represents a conflict of interest in connection with the manuscript.

Corresponding Author:

Renata Corrêa Pascotto

E-mail: renatapascotto@gmail.com

https://doi.org/10.1590/1807-3107bor-2020.vol34.0110

Submitted: Mar 24, 2020

Accepted for publication: July 10, 2020

Last revision: July 20, 2020
Abstract: Acrylic resin has been used in the manufacture of prostheses, however, in the oral cavity, this material starts to retain microorganisms capable of causing gingival inflammation due its porosities. The aim of this study was to evaluate the influence of the use of silicon dioxide as a coating layer applied onto acrylic resin, on the adhesion of Candida albicans $(\mathrm{Ca})$. After the incubation period in Sabouraud Dextrose Broth, a total of $1 \mathrm{ml}$ of the $\mathrm{Ca}$ suspension was added to plate wells, each well containing a specimen of acrylic resin. The adhesion ability of $\mathrm{Ca}$ on acrylic resin was determined by counting colonies. Three groups $(n=6)$ of acrylic resin were assessed: with polishing (RP); without polishing $(\mathrm{RW})$; with polishing and coating layer of silicon dioxide (RPC). $\mathrm{Ca}$ deposited on the surface of the acrylic resin was also observed using Scanning Electron Microscopy (SEM). Statistical assessment by Kruskal-Wallis and Student-Newman-Keuls Method were done $(a=2 \%)$. There was significant difference among the groups. The RPC group showed the lowest growth, with an average of $5.59 \mathrm{Log} \mathrm{CFU} / \mathrm{cm}^{2}$; there was a statistically significant difference in relation to group RW, which presented a growth of $6.07 \mathrm{Log} \mathrm{CFU} / \mathrm{cm}^{2}$ and to group RP with $5.91 \mathrm{Log} C F U / \mathrm{cm}^{2}$ ( $\left.p<000.1\right)$. SEM images demonstrated that in the $\mathrm{RP}$ and RPC group, the surface of the resin had greater regularity, and smaller number of microorganisms. The application of silicon dioxide coating on acrylic resin appears to be a promising alternative, and its use can help in reducing the adhesion of $\mathrm{C} a$ in prostheses.

Keywords: Silicon Dioxide; Acrylic Resins; Candida albicans.

\section{Introduction}

Acrylic resin has been widely used in dentistry in the manufacture of prostheses since 1937, due its several advantages such as low cost and ease of handling. ${ }^{1}$ However, over time, this material retains pigments and microorganisms capable of causing bad breath and gingival inflammation. To date, there is no effective method to reduce the microbial adhesion and pigments retained in the porosities of the acrylic resin surface. ${ }^{2}$ These microorganisms include Candida albicans, which has the ability to adhere onto soft and hard tissues, causing important infections known as candidiasis. ${ }^{3}$

Various cleaning methods have been used for the disinfection of dentures such as toothpastes, vinegar, sodium hypochlorite, 
chlorhexidine gluconate, and chlorhexidine soap. ${ }^{3}$ In spite of acting effectively on biofilm removal from other surfaces, they may change the physical and mechanical properties of resin negatively. ${ }^{4}$ There have been attempts to create acrylic resins with antimicrobial properties by incorporating antifungal agents as in composite resin. ${ }^{5}$ However, these agents can change the properties of the acrylic resin, influencing its aesthetics and durability.

Furthermore, these agents are released over time, reducing their effectiveness. A sealed and flat surface is of great importance in preventing biofilm retention. ${ }^{6}$ Studies have shown that silicon dioxide in nanoscale has been used in hospital environments and does not interfere with surface properties, in addition to minimizing the risk of infection. ${ }^{7}$ Its coating results in a surface with biologic load that is massively reduced and easy to clean. A German patent filed in 2007 by Jürgens and Schwindt, ${ }^{8}$ had already published a set of applications of a nanoscale silicon dioxide coating as well as their use. Furthermore, a recent in vivo study demonstrated the biocompatibility of acrylic resin coated with silicon dioxide promising be a bioprotective coating with low deleterious risk to the individual. ${ }^{9}$ Thus, the objective of this study was to evaluate if the application of a silicon dioxide coating layer on acrylic resin commonly used on temporary partial prosthesis or complete dentures is able to reduce the Candida albicans adhesion.

The null hypothesis was that the use of silicon dioxide as a coating layer on acrylic resin does not act as a retention inhibitor of $C$. albicans adhesion.

\section{Methodology}

\section{Preparation of resin}

Altogether, there were 18 specimens $(10 \times 10 \times 2 \mathrm{~mm}$ dimensions) of acrylic thermoplastic resin (VipiCril Plus, VIPI Indústria Comércio Exportação e Importação de Produtos odontológicos Ltda, São Paulo, Brazil) produced. The acrylic thermoplastic liquid (PALATON, Dencril Comércio de Plásticos Ltda, Pirassununga, Brazil) and the acrylic resin powder (VipiCril Plus, VIPI Indústria Comércio Exportação e Importação de Produtos odontológicos
Ltda, São Paulo, Brazil), were mixed in a container, according to the proportions indicated by the manufacturer. The acrylic resin was inserted into a single rectangular silicone mould $(10 \times 180 \times 2$ $\mathrm{mm}$ dimensions) and after the start of the plastic phase, the material was pressed over 10 minutes in a pneumatic digital polymerizing electric cooker (Protécni Equipamentos, Araraquara, Brazil) at $80^{\circ} \mathrm{C}$ temperature and 100 pounds pressure. The specimens were cut using a double-face diamond wheel in low speed under water cooling to the given dimensions and the finishing was done with a double-face diamond disc (Microdont, São Paulo, Brazil), to obtain standardized dimensions of the specimens. The specimens were randomly divided into three groups ( $\mathrm{n}=6$ each group) according to the surface treatment: with polishing (RP); without polishing (RW); with polishing and coating (RPC). Final polishing for RP and RPC groups, was carried out with a felt disc used with pumice stone and White of Spain (Asper Industria Quimica Ltda, São Caetano do Sul, Brazil).

The coating in the RPC group was a layer of silicon dioxide base (Liquid Glass Shield, TOPTEK Equipment Ltda, Belo Horizonte, Brazil) (RPC), applied according to the manufacturer's instructions. A vial of silicon dioxide (Liquid Glass Shield) was shaken before each application. The silicon dioxide was sprayed on sterile gauze. This gauze was then used to apply to the surface of the resin by rubbing. The resins with silicon dioxide was then left to cure for 24 hours. All groups had a negative control, represented by an uncontaminated composite resin specimen.

\section{Microbial isolates}

This study was conducted with an ATCC 90028 C. albicans. Before the tests, the isolate was reactivated in Sabouraud Dextrose Agar (SDA; Merck \& Co., Inc., Kenilworth, USA), for 24 hours at $37^{\circ} \mathrm{C}$. Briefly, yeasts were subcultured in chromogenic medium CHROMagarTM Candida (Difco, Morgan Hill, USA), to check the purity of the culture after all tests. ${ }^{10}$

\section{Candida albicans growth}

Yeast was grown at $37^{\circ} \mathrm{C}, 120 \mathrm{rpm}$, for 18 hours in Sabouraud Dextrose Broth (SDB; Difco, USA). 
After the incubation period, the suspension was washed in phosphate saline buffer (PBS; pH 7.0) and re-suspended in artificial saliva (Botica Ouro Preto Compounding Pharmacy, Maringa, Brazil) to a final concentration of $1.0 \times 10^{7}$ cells $/ \mathrm{ml}$. A total of $1 \mathrm{ml}$ of this suspension was added to 24 plate wells, with each well containing a specimen of acrylic resin $(1 \times 1 \mathrm{~cm})$ : $\mathrm{RP}, \mathrm{RW}$ and RPC. The plates were incubated for two hours at $37^{\circ} \mathrm{C}$ at $60 \mathrm{rpm} .{ }^{11}$

\section{Quantification of adhesion capacity}

Adhesion capacity were analysed according to Queiroz et al. ${ }^{11}$ with modifications. After the incubation period (2 hours), the suspension of each well containing a specimen was removed and washed once with PBS in order to remove non-adherent yeasts. The specimens were scratched with a needle to remove the adherent yeasts. They were then re-suspended in $1 \mathrm{ml}$ of PBS and a serial and plated dilution in SDA was carried out. These plates were incubated for 24 hours at $37^{\circ} \mathrm{C}$. The number of adherent yeasts was determined by counting the colonies formed and the results were expressed as $\log \mathrm{CFU} / \mathrm{cm}^{2}$.

\section{Scanning electron microscopy}

For the Scanning Electron Microscopy (SEM) analysis, deposits on the surface of the acrylic resin were fixed with glutaraldehyde $(2.5 \%)$ and cacodylate buffer $(0.1 \mathrm{M})$ for 4 hours. Samples were then dehydrated in a series of ethanol washes (70, $80,90,95$, and $100 \%)$. The specimens were fixed on supports, coated with gold-palladium in argon atmosphere, using a gold-sputter module in a highvacuum evaporator. The samples were observed with a SEM (FEI Quanta 250, Hillsboro, USA) at $\times 1000$ magnification.

\section{Statistical analysis}

All tests were performed in triplicate, on three independent days. The results were analyzed using the Software R i386 3.0.2. The data was initially subjected to the Shapiro Wilk test to analyze the data normality. Once a normal distribution was not verified, data were compared with KruskalWallis One-way analysis of variance on ranks and all pairwise multiple comparison procedures
(Student-Newman-Keuls Method) were done. All tests were conducted with $99 \%$ confidence level.

\section{Results}

The average among the duplicates of each group of adherence capacity is represented in Figure 1 and in Figure 2, it is possible to observe the characteristics of the material surface, as well as the adhesion by $C$. albicans, by SEM images.

The RPC group with a layer of silicon dioxide base and polishing showed the lowest growth (5.59 Log $\left.\mathrm{CFU} / \mathrm{cm}^{2} \pm 0.1\right)$, presenting a statistically significant difference $(p<000.1)$ when compared to RW $(6.07 \mathrm{Log}$ $\left.\mathrm{CFU} / \mathrm{cm}^{2} \pm 0.1\right)$ and RP (5.91 Log CFU/ $\left.\mathrm{cm}^{2} \pm 0.1\right)$. This data correlates with the images observed by SEM.

Figure 2 presented a fairly irregular surface on the specimens (RW) as the resin was not polished (Figure 2A) and also shows higher adhesion by $C$. albicans (Figure 2B). However, Figure 2B shows a dense layer on the surface of the acrylic resin of the RW group, probably due to the accumulation of artificial saliva (indicated by the white arrow) with C. albicans (indicated by the red arrows) in the irregularities of the material.

In the groups RP (C and D) and RPC (E and F), the surface of the resin was presented with greater regularity, promoting less adherence by $C$. albicans (indicated by the red arrows).

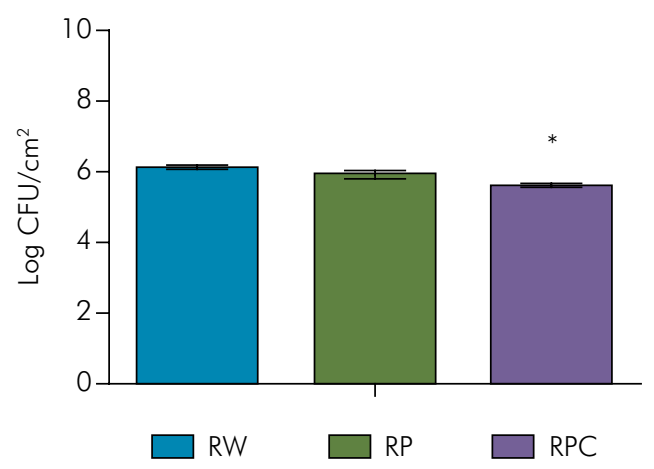

*Student-Newman-Keuls Method. Statistically significant difference $(p<000.1)$.

Figure 1. Median values of the C. albicans adhesion on acrylic resin surfaces according to the three groups tested: RW-without polishing; RP- with polishing; RPC- with polishing and coating layer of silicon dioxide. 

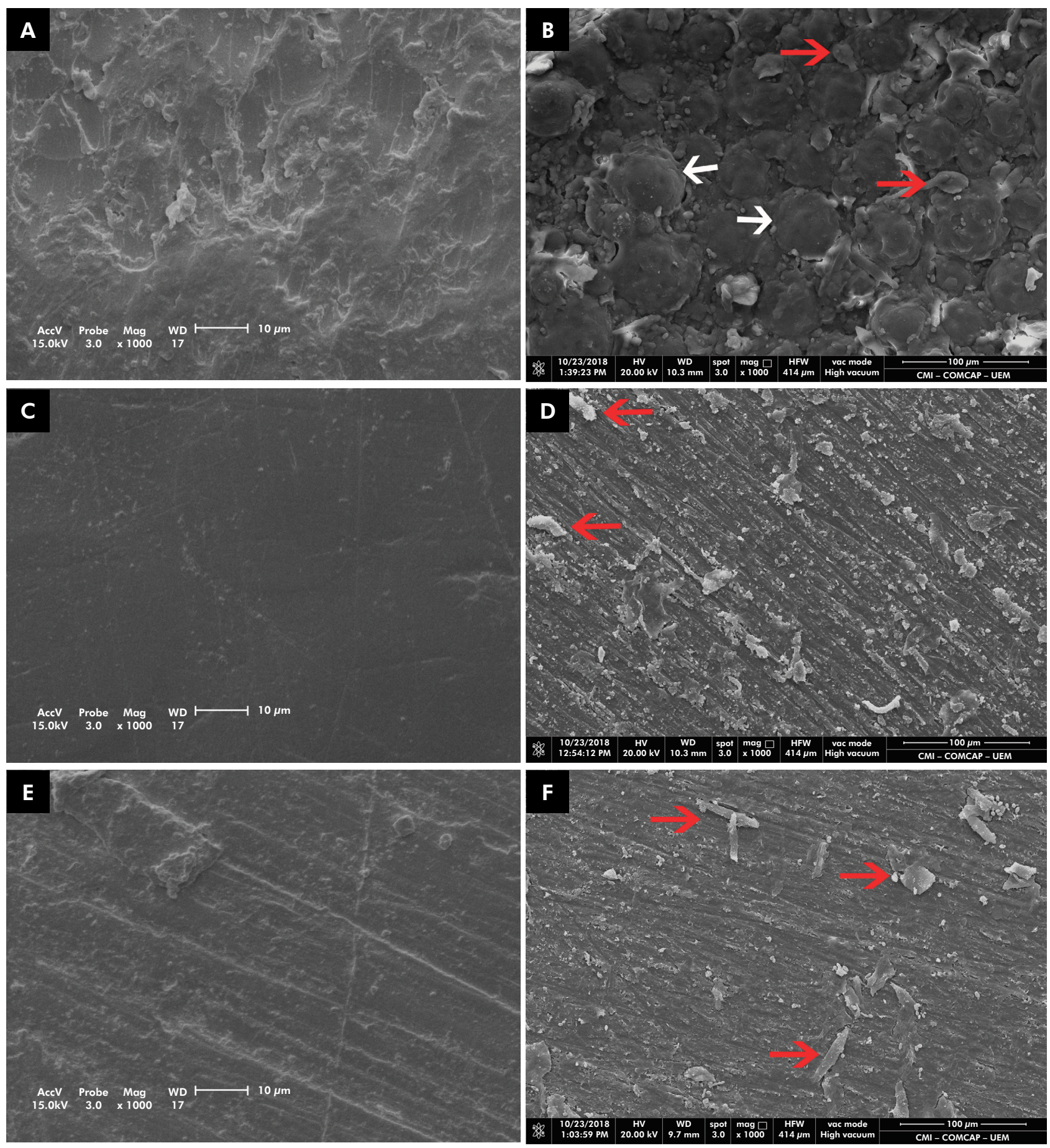

Figure 2. Scanning Electron Microscopy (SEM) analysis of the acrylic resin specimen's surface $(\times 1000$ magnification). (A) Control specimen without yeast on the resin without the polish (RW) surface; (B) Specimen with the yeast on the resin without the polish (RW) surface. White arrows indicate a dense layer probably due to the accumulation of artificial saliva with C. albicans (red arrows) in the irregularities of the material; (C) Control specimen without yeast on the resin polished surface (RP); (D) Specimen with the yeast (red arrows) on the resin polished surface (RP); (E) Control specimen without yeast on the resin polished surface after the use of silicon dioxide coating layer (RPC); (F) Specimen with the yeast (red arrows) on the resin polished surface after the use of silicon dioxide coating layer (RPC). 


\section{Discussion}

In the present research, the null hypothesis was rejected as when silicon dioxide was applied onto polished acrylic resin, the $C$. albicans adhesion was lower than when it was not used, demonstrating that the product has the potential to reduce adhesion of microorganisms on an intraoral prosthesis. This may influence the prevention of local and systemic infections related to the use of prostheses made of acrylic resin. According to clinical studies with Candida spp., counts greater than $100,000\left(10^{5}\right) \mathrm{CFU}$ are related to some infectious process in the oral cavity. ${ }^{10,12,13}$ The present study demonstrated that with a coating of silicon dioxide on acrylic resin there was a reduction in CFU of C. albicans $(<100,000)$.

C. albicans is a commensal yeast, found in the oral cavity of $75 \%$ of the population. Its pathogenicity is greater in immunosuppressed individuals, who can often suffer recurrent infections such as oral candidiasis. ${ }^{3}$ Clinically, these patients present with pain and irritation in the oral mucous membrane. ${ }^{14}$ In an immunosuppressed patient, this infection is closely related to respiratory infections..$^{15}$ These infections are often related to prostheses used by individuals. Acrylic prostheses are commonly used in oral rehabilitation. C. albicans infections are common, especially when patients fail to maintain good hygiene of the prosthesis. This is important because, according to the literature, although patients realise the importance of cleaning a prosthesis, they may not necessarily know how to do it. ${ }^{16}$ In addition, in patients with systemic disorders, candidiasis is more often found in the oral cavity compared to healthy people. ${ }^{17}$

Polished resins that have a coating of silicon dioxide showed the lowest amount of $C$. albicans compared to polished resins and resins without the application of coating, with significant differences between the RPC and RP groups. This demonstrates the usefulness of the coating. The resins with polishing presented results with significant differences in comparison to the resins without polishing; this can be explained by the uneven surface, which can generate more adherence of the microorganism. This was clear with statistical differences between the groups RW vs RP and RW vs RPC.

The ability of $C$. albicans to adhere to acrylic resin surfaces is an important initial step to start colonization of the microorganism and subsequent invasion of the host tissue. ${ }^{3}$ Additional to this, uneven surfaces can increase the accumulation of organisms in prostheses, even after cleaning them. Rough surfaces, with the presence of summits and other particularities, help the growth of hyphae. Prostheses with improper or inadequate polishing present with topology that favors the growth of these hyphae. ${ }^{16}$

Silicon dioxide produces an invisible $<250 \mathrm{~nm}$ thick layer of coating and has been indicated as a bioprotective coating for surfaces susceptible to colonization by microorganisms. ${ }^{8}$ This nanofilm has characteristics such as a cationic nature, resistance to acids, hydrophobia, oleophobia, high flexibility, antibacterial and antifungal properties, resistance to corrosion and abrasion, ${ }^{8}$ and it is possible that these characteristics are responsible for reducing the adhesion of $C$. albicans on the acrylic resin surface coated with silicon dioxide in the present study.

A recent in vivo study testing the biocompatibility of silicon dioxide nanofilm used as an antimicrobial agent on acrylic surfaces, demonstrated that coating with NP-Liquid Glass had the highest level of tissue compatibility, and the best cell repair, being highly promising for use in clinical practice. ${ }^{9}$

In this study, it was not possible to analyze the adherence of silicon dioxide on the surface of the resin, nor the change of surface roughness. Given these limitations, the use of atomic force microscopy would be beneficial in future research, in order to analyze the surface roughness of resins and silicon dioxide adherence on the surfaces. However, the use of a cover layer with silicon dioxide that decreases microbial adhesion appears to be important clinically. Other studies with silicon dioxide should be carried out, to evaluate possible changes on the acrylic resin surface after applying a layer of coating as well as the durability of its protective effect. 


\section{Conclusion}

The application of silicon dioxide coating on acrylic resin seems to be a promising alternative, and its use can help in reducing the adhesion of $C$. albicans in prostheses, which is essential to the longevity and success of treatment with dentures or a removable acrylic resin prosthesis.

\section{Acknowledgments}

This study was supported by Coordenação de Aperfeiçoamento de Pessoal de Nível Superior - Brasil (Capes) - FinanceCode 001, Conselho Nacional de Desenvolvimento Científico e Tecnológico (CNPq) $n^{\circ}$ 421620/2018-8, Fundação de Amparo à Pesquisa do Estado do Paraná (Fundação Araucária) and Financiadora de Estudos e Projetos (Finep/Comcap).

\section{References}

1. Anne G, Oliganti SB, Atla J, Budati S, Manne P, Chiramana S. The effect of aluminum oxide addition on the flexural strength of heat activated acrylic resin: An in vitro study. J Dr NTR Univ Health Sci. 2015;24(1):21. https://doi.org/10.4103/2277-8632.153307

2. Nandal S, Ghalaut P, Shekhawat H, Gulati M. New era in denture base resins: a review. Dent J Adv Stud; $2013 ; 1(3): 136-43$. https://doi.org/10.1055/s-0038-1671969

3. Izumida FE, Moffa EB, Vergani CE, Machado AL, Jorge JH, Giampaolo ET. In vitro evaluation of adherence of Candida albicans, Candida glabrata, and Streptococcus mutans to an acrylic resin modified by experimental coatings. Biofouling. 2014;30(5):525-33. https://doi.org/10.1080/08927014.2014.894028

4. Altieri KT, Sanitá PV, Machado AL, Giampaolo ET, Pavarina AC, Jorge JH, et al. Eradication of a mature methicillinresistant Staphylococcus aureus (MRSA) biofilm from acrylic surfaces. Braz Dent J. 2013 Sep-Oct;24(5):487-91. https://doi.org/10.1590/0103-6440201302289

5. Garcia-Cuesta C, Sarrion-Pérez MG, Bagán JV. Current treatment of oral candidiasis: a literature review. J Clin Exp Dent. 2014 Dec;6(5):e576-82. https://doi.org/10.4317/jced.51798

6. Verran J, Jackson S, Coulthwaite L, Scallan A, Loewy Z, Whitehead K. The effect of dentifrice abrasion on denture topography and the subsequent retention of microorganisms on abraded surfaces. J Prosthet Dent. 2014 Dec;112(6):1513-22. https://doi.org/10.1016/i.prosdent.2014.05.009

7. Mogensen JE, Jørgensen PE, Thomsen TR. A microbiological evaluation of SiO2-coated textiles in hospital interiors: the effect of passive coatings on the cleaning potential of interior textiles. J Ind Text. 2016;46(2):361-71. https://doi.org/10.1177/1528083715580543

8. Jurgens R, Schwindt S, inventors. Google Patents. Anitbakterielle coating composition based on a silica-generating agent, a set of applications, a nanoscale coating the preparation of the coating, further processing of the coating as well as their use. German. DE102006008535A1. 2007 Aug 30.

9. Lacerda-Santos R. Lima ABL, Penha ES, Santos A, Carvalho FG, Pithon MM, et al. In vivo biocompatibility of silicon dioxide nanofilm used as antimicrobial agent on acrylic surface. An Acad Bras Cienc. 2020 Apr;92(1):e20181120. https://doi.org/10.1590/0001-3765202020181120

10. Pieralisi N, Bonfim-Mendonça PS, Negri M, Jarros IC, Svidzinski T. Tongue coating frequency and its colonization by yeasts in chronic kidney disease patients. Eur J Clin Microbiol Infect Dis. 2016 Sep;35(9):1455-62. https://doi.org/10.1007/s10096-016-2684-y

11. Queiroz PA, Godoy JS, Mendonça PS, Pedroso RB, Svidzinski TI, Negri M. Adhesion and biofilm formation in artificial saliva and susceptibility of yeasts isolated from chronic kidney patients undergoing haemodialysis. J Med Microbiol. 2015 Sep;64(9):960-6. https://doi.org/10.1099/jmm.0.000122

12. Bertolini M, Ranjan A, Thompson A, Diaz PI, Sobue T, Maas K, et al. Candida albicans induces mucosal bacterial dysbiosis that promotes invasive infection. PLoS Pathog. 2019;15(4):e1007717. https://doi.org/10.1371/journal.ppat.1007717

13. Hu L, He C, Zhao C, Chen X, Hua H, Yan Z. Characterization of oral candidiasis and the Candida species profile in patients with oral mucosal diseases. Microb Pathog. 2019 Sep;134:103575. https://doi.org/10.1016/j.micpath.2019.103575

14. Yarborough A, Cooper L, Duqum I, Mendonça G, McGraw K, Stoner L. Evidence regarding the treatment of denture stomatitis J Prosthodont. 2016 Jun;25(4):288-301. https://doi.org/10.1111/jopr.12454

15. O’Donnell LE, Smith K, Williams C, Nile CJ, Lappin DF, Bradshaw D, et al. Dentures are a reservoir for respiratory pathogens. J Prosthodont. 2016 Feb;25(2):99-104. https://doi.org/10.1111/jopr.12342 
Silva RVDR, Costa MI, Jarros IC, Del Bel Cury AA, Sidhu SK, Negri M, et al.

16. O'Donnell LE, Alalwan HK, Kean R, Calvert G, Nile CJ, Lappin DF, et al. Candida albicans biofilm heterogeneity does not influence denture stomatitis but strongly influences denture cleansing capacity. J Med Microbiol. 2017 Jan;66(1):54-60. https://doi.org/10.1099/jmm.0.000419

17. Le Bars P, Kouadio AA, N'goran JK, Badran Z, Soueidan A. Relationship between removable prosthesis and some systemics disorders. J Indian Prosthodont Soc. 2015 Oct-Dec;15(4):292-9. https://doi.org/10.4103/0972-4052.171828 